\title{
Supplementary Abstracts of the 15th World Congress of Pharmacology
}

\author{
S4.5 \\ Juvenile animal studies in pediatric drug development: \\ What is the evidence?
}

Anne ZAJICEK, MD, PharmD.

Pediatric Medical Officer, Obstetric and Pediatric Pharmacology Branch, National Institute of Child Health and Human Development, National Institutes of Health, USA

Juvenile animal models have been used to predict pediatric safety, efficacy and pharmacokinetics for a variety of drugs. A series of guidances by inter- nationally recognized regulatory bodies have been developed to address the complexities of extrapolation from juvenile animal to child, including species and corresponding physiologic age of the organ systems of interest. Juvenile animal studies have been predictive of pediatric safety for several agents, including growth inhibition with methylphenidate, methamphetamine and neurobehavioral effects, and changes in sero- tonergic innervation from fluoxetine. The juvenile animal model was not able to predict congenital malformations due to the angiotensin converting enzyme inhibitors, among others. There is consi- derable variability in predictability of animal models, and need for further research to identify mechanisms of toxicity that can be extrapolated across species.

\section{S5.2}

The rationale for application of NSAIDs in Alzheimer's therapy

\section{Sascha WEGGEN, Ph.D.}

Emmy Noether Research Group, Institute of Physiological Chemistry and Pathobiochemistry, Johannes Gutenberg University Mainz, D55128 Mainz, Germany

Non-steroidal anti-inflammatory drugs (NSAIDs) have been considered for treatment and prevention of Alzheimer's disease (AD) for more than two decades. The rationale for this approach was derived from epidemiological studies and from the observation that the causative amyloid pathology in the AD brain is accompanied by a secondary inflammatory response. Given that the primary pharmacological targets of NSAIDs are cyclooxygenases (COX), the reduced expression of inflammatory markers in AD mouse models after peripheral administration of NSAIDs has suggested that these compounds may be beneficial by inhibiting a wide range of inflammatory responses in the central nervous system. Recent findings have shown that NSAIDs possess additional anti- amyloidogenic activities that provide an alternative explanation for their protective effects. Most notably, a subset of NSAIDs has been shown to selectively lower cellular production of the A42 peptide, which appears to be key agent in the pathogenesis of familial and sporadic forms of AD. In contrast to conventional -secretase inhibitors, these -secretase modulators effectively suppress A42 production while sparing processing of NOTCH and other -secretase substrates. Although not fully resolved on the molecular level, the mechanism of action of A42-lowering NSAIDs is independent of COX inhibition and most likely involves direct interaction with components of the -secretase complex or its substrates. Here, we review the available evidence that the efficacy of NSAIDs in AD might be attributable to either anti-inflammatory or anti-amyloidogenic activities. However, we propose that future drug development efforts should focus on the improvement of A42-lowering molecules without COX activity, which could provide a potent means to prevent both amyloid pathology and secondary inflammatory reactions while avoiding clinical sideeffects associated with inhibition of COX and -secretase.

\section{S6.4}

Anti-inflammatory and analgesic aspects of the annexin A1 system

\section{R J FLOWER, S AYOUB, M PERRETTI}

William Harvey Research Institute, Charterhouse Square, London EC1M 6BQ, UK

Annexin A1 is a $37 \mathrm{kDa}$ member of the annexin super-family of proteins. It is widely expressed in cells throughout the body. One intriguing aspect of this protein is that it is glucocorticoid regulated. This regulation occurs at the genomic level and also at the post translational level causing it to be released from cells.

Annexin A1 can bind to FPRL1 receptors and through this can exert a negative inhibitory influence on leukocyte activation and mediator production.

This protein also inhibits hyperalgesia or nociception in inflammatory models. In the latter case at least it appears that the protein has an endogenous inhibitory effect on the synthesis of PGE2 within cells of the spinal cord which are involved in the transmission of the nociceptive stimulus. The Annexin A1 null animal is more susceptible to nociception than the wild type control and this phenotype can be rescued by administration of the protein or the peptide.

RJF is a Principal Fellow of the Wellcome Trust. 


\section{S8.5}

Indoreante, an useful tool to detect several 5-HT-related pharmacological activities

\section{EnriqueHONG}

Dept. of Pharmacobiology, Cinvestav, IPN, Czada de los Tenorios 235, C P 14330, México, D F, Mexico

Indorenate is a 5-HT analogue, possessing a 5-Methoxy group instead of a 5-OH, and a Methyl carboxylate moiety substitution in the carbon. It is a $5-\mathrm{HT}_{1 \mathrm{~A}}$ agonist with central antihypertensive activity, since it decreased blood pressure when given in a lateral brain ventricle or in the left vertebral artery of the cat at lower doses than that decreasing blood pressure by the intravenous route. It also decreased the norepinephrine release from adrenergic nerve terminals by a presynaptic effect. Indorenate is absorbed from the oral route and less than one per cent penetrates into the brain. It decreased blood pressure in renal and spontaneous hypertensive rats, renal hypertensive dogs and also in 21 hypertensive patients with mild or moderate hypertension. It also produced ansiolytic effects in the vogel test and in the burying behavior and antidepressant effects in the rat swimming, and increases the consolidation of memory in the autoshaping test. It also seems to decrease the insulin resistance in SHR. This compound has been very useful to teach research and development activities in an academic institution. This work was partially supported by CONACYT 14473 and 30750M.

\section{S14.4}

\section{PKG and hypoxic pulmonary vasoreactivity}

Yuansheng GAO, Ada D PORTUGALL, Sewite NEGASG, Weilin ZHOU, Lawrence D LONGO, J Usha RAJ

Peking University Health Science Center, Beijing, China; Loma Linda University, School of Medicine, CA, USA; and Harbor-UCLA Medical Center, CA, USA

Cyclic GMP-dependent protein kinase (PKG) plays a critical role in vasodilation induced by cGMP- elevating agents such as nitric oxide, nitrovasodilators, and natriuretic peptides. In the perinatal lungs, cGMP-mediated vasodilation is primarily mediated by PKG. In pulmonary veins of the fetuses, cGMP- induces relaxation, PKG activity, and levels of PKG protein and mRNA are greater after exposed to short term normoxia than hypoxia. The oxygen-dependent modulation of PKG activity may facilitate the postnatal adaptation of pulmonary circulation. In fetal lungs exposed to chronic hypoxia, relaxation of pulmonary arteries induced by cGMP is suppressed. The decreased relaxation is improved by Y27632, an inhibitor of Rho kinase (ROCK). The expression of type II ROCK and the activity of ROCKs are upregulated by chronic hypoxia. An increased ROCK activity may counteract PKG action through its opposing effect on myosin lightchain phosphatase. ROCK inhibitors have been found to be effective in reversing hypoxic pulmonary hypertension in animal models as well as in a clinical study. The restoration of PKG-mediated vasodilation may be one of the underlying mechanisms.

\section{S32.3 \\ Improving quality control of herbal medicine: a biopharma- ceutic/pharmacokinetic approach}

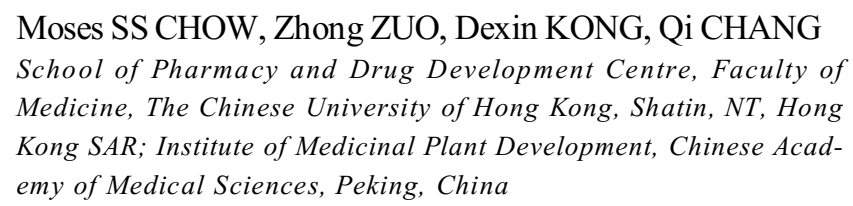

Objective: The aim of this presentation is to propose an in vitro biopharmaceutic/pharmacokinetic (PK) approach which can be suitable for QC of oral herbal products. Methods: The proposed PK approach involves the following steps and was applied to Danshen-Gegen products: (1) identify active chemical components based on in vitro studies; (2) determine content of component in the dosage form; (3) perform in vitro dissolution profile; (4) obtain absorption profile using $\mathrm{CaCO} 2$ cell line; (5) perform metabolic studies of the absorbed components using Phase 1 and Phase 2 liver enzymes; (6) compile a "PK fingerprint" of the active components from about steps. Results: Preliminary data from the study of 2 Danshen-Gegen products showed that the proposed PK approach can identify active components that are capable of exerting biologic activity. The "PK fingerprint" was capable of providing characteristics that can distinguish relevant differences of the 2 products. Conclusion: Our preliminary data indicate that the proposed PK approach in generating the "PK fingerprint" may provide relevant QC measure for herbal products.

Keywords: pharmacokinetics, herbs, quality control

Acknowledgments: Area of Excellence project "Chinese Medicine Research and Further Deve- lopment" (Project No. AoE/B-10/01) coordinated by the Institute of Chinese Medicine of the Chinese University of Hong Kong

\section{CanadaAward Lecture \\ Adipokines: novel pharmacological targets for the treat- ment of obesity related diseases}

Kerry B GORALSKI ${ }^{1,2}$, Tanya C McCARTHY ${ }^{1}$, Elyisha A HANNIMAN $^{1}$, Christopher J SINAL ${ }^{1}$

${ }^{I}$ Deptment of Pharmacology and ${ }^{2}$ College of Pharmacy, Dalhousie University, Halifax, NS, Canada

Obesity is an alarming health problem as this condition is risk factor for type II diabetes and cardiovascular diseases. White adipose tissue secretes a number of signaling peptides (adipokines) including leptin and adiponectin that have 
important endocrine roles in regulating adiposity and metabolism. Increasing evidence also supports a role for adipokines as pathogenic links between obesity and type II diabetes and vascular diseases.

Chemerin is a recently identified proinflammatory cytokine with a role in adaptive and innate immunity. We have identified high-level expression of chemerin, and the cognate receptor chemerinR, in mouse and human adipocytes. This indicates that adipocytes are a source and target for chemerin signaling. Consistent with this, media from cultured adipocytes contained chemerin protein, activated chemerinR signaling in adipocytes and other cell types and stimulated chemotaxis of chemerinR expressing cells. Targeted knockdown of chemerin or chemerinR expression in preadipocytes blocked adipogenesis while knockdown in mature adipocytes affected glucose and lipid homeostasis in these cells.

Our finding that chemerin is a novel adipokine with a regulatory role in adipogenesis and metabolism identify the importance of this peptide in adipose tissue biology and as a potential therapeutic target for treatment of metabolic disorders.

\section{S27}

Levels of anandamide and oleoylethanolamide in intestines of food-deprived rats are regulated through their precursors

GittePETERSEN ${ }^{1}$, Camilla SORENSEN ${ }^{1}$, Patricia CSCHMID $^{2}$, Andreas ARTMANN ${ }^{1}$, Mads TANG-CHRISTENSEN ${ }^{3}$, Steen HHANSEN $^{4}$, Philip J LARSEN ${ }^{3}$, HaraldHOSCHMID ${ }^{2}$, Harald S HANSEN $^{1}$

${ }^{1}$ Deptment Pharmacol. Pharmacother., and ${ }^{4}$ Deptment Pharmacy \& Analyt Chem, The Danish University of Pharmaceutical Sciences, 2100 Copenhagen, Denmark, ${ }^{2}$ The Hormel Institute, University of Minnesota, MN 55912, USA, ${ }^{3}$ Rheoscience, 2610 Rodovre, Denmark

The anorectic lipid oleoylethanolamide (OEA) and the orexigenic lipid anandamide both belong to the group of $N$ acylethanolamines (NAEs) that are generated by the enzyme $N$-acyl-phosphatidylethanolamine (NAPE)-hydrolyzing phospholipase D (NAPE-PLD). The previously reported diverging levels of the two bioactive lipids during starvation were investigated in rat intestines after $24 \mathrm{~h}$ of starvation as well as after re-feeding. Total levels of NAPEs and NAEs were decreased upon starvation whereas the level of the anandamide precursor-NAPE was significantly increased. The level of 2-arachidonoyl-glycerol was unchanged as was the activity of $N$-acyltransferase, NAPE-PLD, and fatty acid amide hydrolase (FAAH) upon starvation and re-feeding. In conclusion, remodeling of the amide-linked fatty acids of NAPE is responsible for the opposite effects on levels of anandamide and OEA in intestines of food-deprived rats and not a change in NAPE-PLD or FAAH, or an alternative biochemical route for anandamide synthesis.

Key words: oleoylethanolamide; anandamide; appetite; fatty acid remodeling

\section{P030061}

The chemopreventive effects of bicyclol on hepato-carcinogenesis in vitro and in vivo

\author{
Hua SUN, Geng-tao LIU* \\ Institute of Material Medica, Chinese Academy of Medical Sciences, \\ Beijing, China
}

It is well known that the most frequently causes of human hepatocellular carcinoma are chronic hepatitis virus infections. If an anti-hepatitis drug has suppressing effect on the development of hepatocarcinogenesis, this kind of drug would be of great clinical value. Bicyclol, a novel antihepatitis drug, has been licensed to treat HBV infection clinically. The aim of this study was to investigate the chemopreventive effect of bicyclol and its mechanism on hepatocarcinogenesis in vitro and in vivo. The methods and results are as follows: WB-F344 rat liver epithelial cells were chemically transformed by 3 -methylcholanthrene and 12-O-tetradecanoyl-phorbol-13-acetate in vitro, bicyclol at non-cytotoxic doses obviously inhibited the malignancy of WB-F344 cells. In the twostage model of hepatocarcinogenesis in mice with diethyl nitrosamine as the initiator and phenobarbital as the promotor, oral administration of $200 \mathrm{mg} /$ $\mathrm{kg}$ and $100 \mathrm{mg} / \mathrm{kg}$ of bicyclol showed a significant inhibition of the incidence of the liver tumors. The model control mice developed 33.3\% HCC and 55.6\% hepatoma at Week 20, while the mice in bicyclol $200 \mathrm{mg} / \mathrm{kg}$ group showed no tumor in livers. The mechanisms of bicyclol are multiple.

\section{P060180}

Neurochemical and behavioural actions of a novel dopamine $D_{3} / D_{2}$ receptor antagonist/partial agonist putative antipsychotic

Kiss B, Gyertyán I, Sághy K, Laszlovszky I, Horváth A, Laszy J, Schmidt É, Szabó Gy, Domány Gy, Ágainé Csongor É, Adham N*',Tihanyi K, Szombathelyi Zs

Pharmacological and Drug Safety Research, Gedeon Richter Ltd., Budapest. *Forest Research Institute, Jersey City, NJ, USA

We report the pharmacological activity of an RG compound that has been developed on the hypotheses that $\mathrm{D}_{2}$ receptor antagonism is required for antipsychotic activity and that amplified $D_{3}$ receptor antagonism may carry favourable additional effects such as improved cognition and fewer extrapyramidal symptoms.

The molecule displayed subnanomolar in vitro affinity at human $\mathrm{D}_{3}$ receptors, approximately six- and thirty-times greater affinity than for human $\mathrm{D}_{2}$ and $5-\mathrm{HT}_{1 \mathrm{~A}}$ receptors, respectively. It increased dopamine turnover in mouse striatum and olfactory tubercle with notable limbic preference. In 
reserpine-treated mice it showed partial agonistic effect, ie, it returned the enhanced biosynthesis rate of dopamine to normal level. The compound displayed high in vivo potency, comparable to risperidone, in rodent models predictive of antipsychotic activity, including the inhibition of apomorphine-, amphetamine- and phencyclidine-induced behaviours as well as conditioned avoidance response. It showed no cataleptogenic activity at doses that were $>100$ fold the pharmacologically effective dose. The RG compound restored the impaired learning performance of scopolaminetreated rats in a water-labyrinth learning paradigm.

The results demonstrate that highly potent $D_{3}$ receptor antagonist activity may add favourable components (beneficial cognitive effects, low risk of EPS liability) to the activity profile of a potent $\mathrm{D}_{2}$ antagonist. The antipsychotic character of this compound clearly differs from atypical antipsychotics with $\mathrm{D}_{2} / 5-\mathrm{HT}_{2 \mathrm{~A}}$ receptor antagonist activity.

\section{P110116}

Prevent effects of dammonii glycyrrhizinatis on focal cerebral ischemia-reperfusion injury in rats

\author{
Ya-jun LIU ${ }^{1 *}$, Ji-liang WU ${ }^{2}$ \\ ${ }^{I}$ School of Medicine, Wuhan Polytechnical University; ${ }^{2}$ School of \\ Medicine, Xianning College, China
}

Objective: To study the prevent effects of Dammonii Glycyrrhizinatis (DG) on focal cerebral ischemia-reperfusion injury in rats. Methods: male wistar rats were randomized into:sham group, ischemiareperfusion group (IR), DG group $(20 \mathrm{mg} / \mathrm{kg})$. There were 8 animals in each group. The animal models were estabilished by middle cerebral artery occlusion (MCAO). At $12 \mathrm{~h}$ and $24 \mathrm{~h}$ after MCAO for $2 \mathrm{~h}$, the content of malondiadehyde (MDA) and superoxide dismutase (SOD) activity, ultrastructure of brain tissue, neuron cell apoptosis, the protein expression of Bcl-2 gene, intercellular adhesion molecule-1 (ICAM-1) mRNA expression and neutrophil infiltration were estimated respectively. Results: Compared with IR group, MDA content, the pathological damage of brain tissue, neuron cell apoptosis index, ICAM-1 mRNA positive vasa, neutrophil infiltration were significantly decreased, reversely the activities of SOD and the protein expression of Bcl-2 gene upregulated markedly in DG group. Conclusion: DG can protect brain tissue against focal cerebral ischemiareperfusion injury.

Key words: DG; focal cerebral ischemia; apoptosis; gene expression

\section{P110245}

About the possible mechanisms of cerebral blood flow im-
pairment in early hypokinesia

Viulen P HAKOBYAN ${ }^{1}$, Armen H MANUKYAN ${ }^{2}$, Aram V

\section{BAYKOV $^{3}$}

${ }^{1-3}$ Department of Pharmacology, Yerevan State Medical University, Koryun Street 2, Yerevan, 375025, Republic of Armenia

The experiments were performed on mongrel rats and dogs. An ability of different drugs and compounds to change the amplitude of isotonic contractions of isolated vascular rings (IVR) as well as cerebral blood flow (CBF) have been estimated in early hypokinesia (HK). The concentrations of malonic dialdehyde (MDA) and acylhydroperoxydes (AHP) in plasma and erythrocyte membranes have been estimated. Lipid peroxidation (LPO) process intensification is noted in HK with MDA and AHP accumulation. Our previous investigations testify to a vasoconstrictor activity of MDA and its ability to increase IVR contraction. Besides, an increase of the sensitivity of the vessels to the endogenous vasoconstrictor agents is noted. The results of current work testify to a decrease in the sensitivity of IVR to papaverine, drotaverine and caffeine. Taking into account the results of current and previous works, we can conclude, that HK changes the sensitivity of the cerebral blood vessels to the endogenous vasoconstrictor and vasodilator agents. Thus, CBF decrease in early HK can be stipulated by LPO product accumulation and the vascular sensitivity change to the different vasoactive agents.

Key words: hypokinesia, vasoactive agents, $\mathrm{CBF}$

\section{P300030}

Protective effects of guattegaumerine on hydrogen peroxide-induced injury of cultured cortical cells

\author{
Qing LÜ, Min ZHENG*, Lian-jun GUO* \\ Department of Pharmacology, Tongji Medical College, Huazhong \\ University of Science \& Technology, Wuhan, China
}

To investigate the protective effects of Guattegaumerine (Gua) on cortical cells against hydrogen peroxide $\left(\mathrm{H}_{2} \mathrm{O}_{2}\right)$ induced injury. MTT method was used to assess cell viability and LDH leakage to evaluate cell injury. TAOC, GSH, SOD activity and MDA content were measured to evaluate cell antioxidant ability. Results showed that after treatment with $6.25 \mu \mathrm{mol} / \mathrm{L} \mathrm{H}_{2} \mathrm{O}_{2}$ for 24 hours, cell viability was significantly reduced to $26 \pm 6 \%$ and $\mathrm{LDH}$ leakage was increased to $178.6 \pm 35.0 \mu / \mathrm{L}$ compared with normal group of $97.5 \pm 22.0 \%$ and $56.5 \pm 2.0 \mu / \mathrm{L}$, respectively. $2.5 \mu \mathrm{mol} / \mathrm{L}$ Gua pretreatment markedly improved cell viability to $56.0 \pm 23.9 \%$ and reduced LDH activity to $129.7 \pm 63.4 \mu / \mathrm{L}$, respectively. T-AOC and SOD activity of cells treated with $\mathrm{H}_{2} \mathrm{O}_{2}$ were decreased to $1.51 \pm 0.6 \mu / \mathrm{mL}$ and $8.5 \pm 1.2 \mu / \mathrm{mL}$, respectively, and MDA content was increased to $6.8 \pm 0.3 \mu \mathrm{mol} / \mathrm{L}$ compared with normal cells. $2.5 \mu \mathrm{mol} / \mathrm{L}$ Gua pretreatment could increase T-AOC and SOD activity to $2.6 \pm 0.8 \mu / \mathrm{mL}$ and $9.5 \pm 1.5 \mu / \mathrm{mL}$, and decrease MDA content to $6.1 \pm 0.2 \mu \mathrm{mol} / \mathrm{L}$ compared with $\mathrm{H}_{2} \mathrm{O}_{2}$ group. The results suggested that Gua may protect cortical neurons from $\mathrm{H}_{2} \mathrm{O}_{2}$-induced injury by improving cell antioxi- 
dant ability.

Key words: guattegaumerine; cortical cells; hydrogen peroxide

\section{P360084}

Adrenoceptor mediated phosphorylation of AMPK differs between brown adipose tissue and skeletal muscle

Hutchinson DS ${ }^{1,2}$, Chernogubova $\mathrm{E}^{2}$, Cannon $\mathrm{B}^{2}$, Bengtssion $\mathrm{T}^{2}$ ${ }^{1}$ Department of Pharmacology, Monash University, Victoria 3800 , Australia \& ${ }^{2}$ Department of Physiology, Wenner-Gren Institute, Arrhenius Laboratories F3, Stockholm University, SE10691 Stockholm, Sweden

AMP-activated protein kinase (AMPK) is a heterotrimeric protein that acts as a sensor of cellular energy homeostasis in adipose and muscle cells. Activation of AMPK activates catabolic pathways (ie glucose uptake (GU), glycolysis and $\beta$-fatty acid oxidation) and inhibits anabolic pathways (i.e. fatty acid and cholesterol synthesis) through interactions with metabolic enzymes and proteins and effects on gene expression. Adrenoceptor (AR) stimulation causes increases in GU primarily by $\beta_{3}$-ARs in brown adipocytes and by both $\beta_{2}$ - and $\alpha_{1}$-ARs in L6 skeletal muscle cells and this study investigated if AMPK is involved in AR-mediated increases in glucose uptake. Immunoblotting using specific antibodies for p-AMPK and total AMPK were performed and GU was measured. In brown adipocytes AMPK was phosphorylated only by stimulation of $\beta_{3}$-ARs, and this effect was mimicked by forskolin. The AMPK inhibitor compound $\mathrm{C}$ partially inhibited GU mediated by $\beta_{3}$-ARs. In L6 cells $\alpha_{1}$ ARs, but not $\alpha_{2}$ - or $\beta_{2}$-ARs, phosphorylated AMPK and GU in response to $\alpha_{1}$-ARs was inhibited by compound $C$ while GU mediated by $\beta_{2}$-ARs was not. In conclusion, AMPK is phosphorylated by different AR subtypes in brown adipocytes and skeletal muscle.

\section{P360085}

Distinct pathways regulate endothelium derived hyperpolarising factor-mediated responses in rat mesenteric arteries

Inmaculada C VILLAR ${ }^{1}$, Amrita AHLUWALIA ${ }^{1}$, Adrian J HOBBS $^{2}$

${ }^{l}$ Clinical Pharmacology, Barts \& The London, Charterhouse Square, London, EC1M 6BQ; ${ }^{2}$ Wolfson Institute for Biomedical Research, University College London, Gower Street, London, WC1E 6AE, UK

Endothelium-derived hyperpolarising factor (EDHF) hyperpolarises and dilates vascular smooth muscle and plays a key role in regulating local blood flow. To produce effective blockade of both EDHF release (from endothelial cells) and hyperpolarisation (in smooth muscle cells) it is necessary to use specific inhibitor combinations. Thus, we hypo-thesised that distinct pathways may exist to regulate the biological activity of EDHF(s). EDHF-mediated relaxations of rat mesenteric arteries were inhibited by the $\mathrm{IK}_{\mathrm{Ca}}$ blocker TRAM$34(10 \mu \mathrm{mol} / \mathrm{L})$ in combination with the $\mathrm{K}_{\mathrm{IR}}$ inhibitor $\mathrm{Ba}^{2+}(30$ $\mu \mathrm{mol} / \mathrm{L} ; P<0.001)$. The $\mathrm{Na}^{+} / \mathrm{K}^{+}$-ATPase inhibitor ouabain (1 $\mathrm{mmol} / \mathrm{L})$ alone partially inhibited EDHF-induced relaxation $(P<0.001)$, but no additional blockade occurred when combined with TRAM-34 ( $P>0.05)$. However, ouabain in combination with the $\mathrm{SK}_{\mathrm{Ca}}$ blocker apamin $(100 \mathrm{nmol} / \mathrm{L})$ further inhibited the EDHF response when compared to ouabain alone $(P<0.01)$. These data reveal that two distinct pathways regulate the biological activity of EDHF in rat mesenteric resistance arteries and intimate the existence of at least two EDHFs.

Supported by The British Heart Foundation and The Wellcome Trust

Key words: endothelium-derived hyperpolarizing factor, vasodilatation

\section{P110117}

Hesperetin inhibits rat aortic vascular smooth muscle cells proliferation by upregulation of p27kip1

Yong-Ri JIN ${ }^{1}$, Ji-Yeon YU ${ }^{1}$, Ju-Hyun LEE ${ }^{1}$, Yong LIM ${ }^{1}$, Tack-Joong $\mathrm{KIM}^{1}$, Yong-He ZHANG ${ }^{2}$, Hwan-Soo YOO ${ }^{1}$, Yeo-Pyo YUN ${ }^{{ }^{*}}$

${ }^{I}$ College of Pharmacy, Research Center for Bioresource and Health, Chungbuk National University, Cheongju 361-763, Korea; ${ }^{2}$ Department of Pharmacology, School of Basic Medical Science, Peking University, Beijing 10083, China

In this study, we have investigated the effect of hesperetin on the PDGF-BB-induced proliferation of primary cultured rat aortic VSMCs. Hesperetin significantly inhibited $50 \mathrm{ng} /$ mL PDGF-BB-induced VSMCs proliferation and $\left[{ }^{3} \mathrm{H}\right]$ thymidine incorporation into DNA at concentration of 5, 25, 50, and $100 \mu \mathrm{mol} / \mathrm{L}$. In accordance with these findings, hesperetin revealed blocking of the PDGF-BB-inducible progression through $\mathrm{G} 0 / \mathrm{G} 1$ to $\mathrm{S}$ phase of the cell cycle in synchronized cells. Western blot showed that hesperetin inhibited phospholylation of retinoblastoma protein and cyclin A, cyclin D, cyclin E, CDK2 as well as PCNA expressions, and restored downregulation of CKI p27kip1, while did not affect CKI p27waf1, p16INK4, p53, and CDK4 expressions as well as early signaling transduction such as ERK1/2, Akt, p38, JNK, and PDGF-R $\beta$ phospholylation. These results suggest that hesperetin inhibits PDGF-BB-induced rat aortic VSMCs proliferation via G0/G1 arrest in association with the downregulation of the expression of retinoblastoma protein and cycin A, cycin D, cycin E, CDK2 as well as PCNA proteins, and upregulation of $\mathrm{p} 27 \mathrm{kip} 1$, which may begin to explain the proposed health benefits of high hesperetin intake. 